\title{
Cochran's Q-Test on Soil Helminth Prevalence
}

\author{
I. J. David ${ }^{1}$, M. U. Adehi ${ }^{2}$, P. O. Ikwuoche ${ }^{1}$ \\ ${ }^{1}$ Department of Mathematics and Statistics, Federal University Wukari, Nigeria, \\ e-mail: davidij@fuwukari.edu.ng \\ ${ }^{2}$ Department of Statistics, Nasarawa State University, Keffi, Nigeria
}

\begin{abstract}
SUMMARY
A study was made of the prevalence of nine geohelminth egg types in 184 soil samples from 16 recreational parks in Abuja metropolis, Nigeria. Cochran's Q-test was applied to determine whether the difference in the proportions of the egg types found in the soil samples was significant. At a 5\% significance level, it was found that the prevalence of the egg types was significantly different in the 184 soil samples from 16 parks. To identify which of the geohelminth eggs had a significantly larger mean proportional prevalence, a minimum required difference mean comparison technique was applied. The mean comparison test showed that Taenia and Coccocidia eggs were highly prevalent, with significantly larger mean proportions than the other analyzed geohelminth eggs in the 184 examined soil samples.
\end{abstract}

Keywords: Cochran's Q-test, Minimum required difference, Geohelminth eggs, Soil, Recreational parks

\section{Introduction}

Helminth infections are the most common parasitic infections in humans, with the highest prevalence in tropical countries. Due to provision of food, where invertebrate vectors such as insects and molluscs flourish, and unhygienic conditions, food becomes infected with parasite eggs (Lindquist and Cross, 2017). According to Galaktionov (2017), helminths are a group of worms that are habitually parasitic in birds, and they include flatworms, further subdivided into cestodes (tapeworms) and trematodes (flukes), as well as nematodes (roundworms) and acanthocephala (thornyheaded worms). Also, Khana et al. (2019) noted that some species of seabirds often come into interaction with 
humans (harvested species or gulls), and thus may be a basis for zoonotic disease. However, Pal and Chakravarty (2020) made it clear that helminths do not reproduce within a host; hence, they differ from protozoa. Thus, the number of helminths existing in an individual is equal to the number that found entry into it. Most animals host few worms, but a few host a great number of worms. The magnitude of the parasitic load in a host is controlled by genetic features and the host's response to these parasites. Helminths are easily diffused to humans through food, water, soil, and arthropod and molluscan vectors. They can infect every organ and organ system, including the liver, lungs and blood; they have high prevalence in the intestines, and occasionally the brain and other organs (Lindquist and Cross, 2017). Many studies have demonstrated that helminth infections lower the risk of autoimmunity or allergy. For instance, an inverse correlation between autoimmune liver diseases and Strongyloides stercoralis infection was demonstrated in Okinawa, Japan (Aoyama, 2007).

Cross-sectional studies on the relationship between skin prick tests and helminth infections suggested a general protective effect on the atopic reaction (Flohr et al., 2009). Those authors summarized the effects of geohelminths on the risk of asthma, according to previous studies: hookworm lowered the risk of asthma, while Ascaris increased the risk and Trichuris had no effect. Overall, it is concluded that at least some helminths seem to have anti-allergic or antiinflammatory effects in humans. A disproportionate burden of helminthiases in human populations occurs in marginalized, low-income, and resourceconstrained regions of the world, with over 1 billion people in developing areas of sub-Saharan Africa, Asia, and the Americas infected with one or more helminth species. The morbidity caused by such infections imposes a substantial burden of disease, contributing to a vicious circle of infection, poverty, decreased productivity, and inadequate socioeconomic development. Furthermore, helminth infection accentuates the morbidity of malaria and HIV/AIDS, and impairs vaccine efficacy (Lustigman, 2012). Soil transmitted helminth (STH) infections remain a significant health problem in resource-limited countries due to difficulties in the implementation of control measures. In Nigeria, for instance, 
despite several community-based and provincial reports, national data on prevalence, burdens and risk zones (RZs) for STH infections are lacking (Karshima, 2018).

Recently, Ngwese et al. (2020) stated that globally about 1.5 billion people are infected with STHs worldwide. Of these, 270 million are preschool children and over 568 million are school-aged children who require treatment and prevention interventions. People hosting heavy infections have a higher morbidity, while people carrying low-intensity infections are typically asymptomatic. The most susceptible groups are predominantly children of school age between the ages of 5 and 15 years, as well as pregnant women (Bethony et al., 2006; Goodman et al., 2007; Adegnika et al., 2007). Infections are greater in tropical and endemic countries with poor hygienic environments, limited potable water and restricted healthcare facilities (WHO, 2002; Bethony et al., 2006; Lammie et al., 2006). The risk of infection is greater in farmers during their monotonous agricultural work, and women and children during their domestic and recreational activities, where they come in contact with polluted water (WHO, 2002).

Karshima (2018) used Preferred Reporting Items for Systematic Reviews and Meta-Analyses (PRISMA) to determine the prevalence, distribution and RZs for STH infections among Nigerian children through a meta-analysis of data published between 1980 and 2015. A pooled prevalence estimate (PPE) was determined by the random-effects model, while heterogeneity was evaluated using Cochran's Q-test. They examined a total of 34,518 children aged 0-17 years across 19 Nigerian states, of whom 18,901 were infected with one or more species of STH. They found an overall PPE for STH infections of 54.8\% (95\% CI: 54.2-55.3) with sub-group PPEs ranging from 13.2\% (95\% CI: 11.5-15.1) to 80.9\% (95\% CI: 80.0-81.7). The highest PPEs for STH infections were observed among children within community settings $(59.0 \%$, 95\% CI: 57.7-60.4) and school-aged children (54.9\%, 95\% CI: 54.3-55.5). Also, they found that Ascaris lumbricoides was the most prevalent species (44.6\%, 95\% CI: 44.0-45.2). Over $36 \%(15 / 41)$ of the studies published are from south-western Nigeria, making it 
the only high-risk zone (HRZ) for STH infections; the other regions are classified as low-risk zones (LRZs). Isaac et al. (2019) studied the prevalence of STHs in primary school playgrounds in Edo state, southern Nigeria, for both the wet and dry seasons. They used ANOVA to test differences in monthly variation, and the Student $t$-test to compare variations between seasons for prevalence and parasite density data. Post hoc analysis using the Newman-Keuls multiple comparison test was applied to data on monthly Strongyloides density for the dry season. Differences in mean values were significant at $p<0.05$. Their findings showed that out of the six parasites found in both seasons, Ascaris was preponderant in the dry season, followed by Strongyloides and hookworm. In the wet season, Strongyloides was predominant, followed by Ascaris and hookworm. The frequency of occurrence of hookworm in the wet season was larger than in the dry season, and Trichuris parasites were comparatively infrequent in both seasons.

According to Vickers (2005), the reason for recommending nonparametric over parametric techniques, unless certain conditions are met, is often not made obvious. But techniques for statistical inference from randomized trials can fail only in one of two ways, that is, they can inappropriately reject the null hypothesis of no difference between groups (false positive, type I error) or inappropriately fail to reject the null hypothesis (false negative, type II error). Data type is also of concern in parametric and nonparametric techniques. Nominal data which has no explicit pattern, no numerical significance and is differentiated by a naming system is often not normally distributed, and it is typically suitable for most nonparametric methods. Also, ordinal data which has a specific order on the scale of measurement with limiting numerical meaning is mostly analyzed using nonparametric techniques. However, interval and ratio scaled data are often analyzed with parametric methods, except when the assumption of normality is not met even after transformation (Vonk, 2011). In this setting, Cochran's Q-test (CQT) is a typical nonparametric technique for handling binary responses, that is, nominal responses measured two or more times from the same sample (or from two or more samples that have been matched to one or additional vital variables). 
In another approach, two or additional comparable qualitative variables are measured from an identical sample (typically at equal times). In both applications, the CQT is used to compare the distributions of the two or more qualitative variables. The CQT is an extension of McNemar's test enabling more variables to be handled (Sheskin, 2011). The CQT was applied in a study of the detection of heterogeneity in meta-analyses (Pereira, 2010) and it was found that the CQT's power is low in detecting between-study variance in some cases, when 1011 meta-analyses of clinical trials with $\geq 4$ studies and binary outcomes were evaluated. Okeh et al. (2016) compared the CQT with a modified chi-square, $\chi^{2}$ statistic using data comparing four different types of drugs on 60 patients grouped into blocks $(4 \times 15)$. They found that their modified $\chi^{2}$ statistic was less powerful than the CQT. In this research, soil contamination with geohelminth eggs in 16 different recreational parks in Abuja, Nigeria was assessed using the CQT to determine whether the presence $[+]$ or absence $[-]$ of the contaminants were equally distributed across the recreational parks.

\section{Materials and methods}

This section presents the method of soil sample collection and processing of the samples in the laboratory for determining the presence or absence of geohelminth eggs. Finally, the CQT procedure for data analysis is presented.

\subsection{Soil sample collection and processing}

Samples were collected in the months from September to December 2019. Five long strides of the researcher were used to measure an area of approximately $25 \mathrm{~m}^{2}$. At every $2 \mathrm{~m}^{2}$, a mark was made and a number placed. The marked areas were selected by balloting and replacement, where ballot papers with the names of all twenty parks were placed together in a container and picked randomly, listed and replaced into the container. To collect a sample, the marked site was dug to about $3 \mathrm{~cm}$ deep using a hand trowel, after which about $100 \mathrm{~g}$ of soil was collected. The collected samples were then stored in sealed labeled polythene 
bags and set aside in the dark prior to examination as described by Maikai (2008). The samples were then transported by road to the laboratory in Zaria, a journey of about 4 hours.

Collected samples were taken for $\mathrm{pH}$ and texture analysis at the Soil Science Laboratory of the Institute for Agricultural Research (IAR) of Ahmadu Bello University, Zaria, using the method described by Page et al. (1982). In the laboratory, egg recovery was performed by the method described by Maikai (2008), which is a modified version of the sieving method devised by Ruiz de Ybanez et al. (2000). Briefly, $50 \mathrm{~g}$ of soil was weighed, mixed in a plastic container and washed with running tap water into a $250 \mathrm{ml}$ beaker, using three sieves with mesh diameters $100 \mu \mathrm{m}, 400 \mu \mathrm{m}$ and $850 \mu \mathrm{m}$,. The filtrate was left to settle for $15 \mathrm{~min}$ and the liquid decanted. The sediment was collected in $30 \mathrm{ml}$ centrifuge tubes, shaken with $15 \mathrm{ml}$ zinc sulphate (specific gravity 1.25) floatation solution, and centrifuged at $1500 \mathrm{x}$ g for $5 \mathrm{~min}$. The supernatant was then poured into dropper bottles according to the sample and park. These were used to fill two chambers of McMaster slides. The slides were allowed to settle for $5 \mathrm{~min}$, and were then examined under a light microscope $(\times 10)$ for the presence of geohelminth eggs. Two drops of supernatant were also placed on a slide to view the morphological characteristics of eggs as described by Soulsby (1987), which were used to identify the eggs.

\subsection{Cochran's Q-test}

Suppose that binary responses $\left\{X_{i j}, j=1,2, \ldots, c ; i=1,2, \ldots, n_{j}\right\}$ are recorded on units exposed to $c$ environments $\left\{E_{j}\right\}$. Let $X_{i j}=1$ denote a positive response to $E_{j}$ and let the expected values $\mathrm{E}\left(X_{i j}\right)=\boldsymbol{\Delta}_{j}, j=1,2, \ldots, c$. The obvious interest here is to use the observed data $\left\{x_{i j}\right\}$ to test the hypotheses

$$
H_{0}: \Delta_{l}=\Delta_{2}=\ldots=\Delta_{c} v s . H_{l}: \Delta_{i} \neq \Delta_{j} \text { for at least one pair }(i, j) .
$$

In this study, the $c$ environments are the different geohelminth egg types, and our interest is in comparing the proportions of positive $\left(X_{i j}=1\right)$ responses to these egg types. It is important to consider how to choose the appropriate method to 
perform this test by specifying how the data were obtained. There are two cases that always need to be considered. In Case $\mathbf{I}, X_{i l}$ and $X_{j k}$ are mutually independent if $(i, l) \neq(j, k)$. In Case II, $X_{i l}$ and $X_{i k}(i=1, \ldots, r)$ are recorded on the same unit, and therefore are typically not independent. Case II is referred to as an issue of equality testing of correlated proportions (Gayle, 2010). The data structure in Case $\mathbf{I}$ is in the form of a completely randomized, one-way design with $n_{j}$ binary responses in environment $E_{j}, j=1,2, \ldots, c$, as set out in Table 1 .

Table 1. Case I, $\left\{X_{i j}\right\}$ independent

\begin{tabular}{cccc}
\hline \multicolumn{4}{c}{ Environments } \\
\hline $\operatorname{Egg}_{1}$ & $\operatorname{Egg}_{2}$ & $\ldots$ & $\operatorname{Egg}_{c}$ \\
\hline$X_{11}$ & $X_{12}$ & $\cdots$ & $X_{1 c}$ \\
$X_{21}$ & $X_{22}$ & $\cdots$ & $X_{2 c}$ \\
$\cdots$ & $\cdots$ & $\cdots$ & $\cdots$ \\
$X_{n_{1} 1}$ & $X_{n_{2} 2}$ & $\cdots$ & $X_{n_{c} c}$ \\
\hline
\end{tabular}

In Case II, each of $r$ units is exposed to all $c$ environments. The exposures to the environments are independently randomized over each unit. This data structure is a randomized complete block design where the units are blocks and the responses are binary. In this research, Case II is characteristic for our data structure, implying correlated proportions, that is, not independent. The reason is that in each recreational park soil sample collected, all geohelminth eggs were checked for presence or absence. Case II has the advantage of controlling heterogeneity among units. Table 2 presents the layout of the design.

Table 2. Case II, correlated proportions

\begin{tabular}{cccccc}
\hline Blocks & $\operatorname{Egg}_{1}$ & $\operatorname{Egg}_{2}$ & $\ldots$ & $\operatorname{Egg}_{\mathrm{c}}$ & Row total \\
\hline 1 & $\mathrm{X}_{11}$ & $\mathrm{X}_{12}$ & $\cdots$ & $\mathrm{X}_{1 \mathrm{c}}$ & $\mathrm{R}_{1}$ \\
2 & $\mathrm{X}_{21}$ & $\mathrm{X}_{22}$ & $\ldots$ & $\mathrm{X}_{2 \mathrm{c}}$ & $\mathrm{R}_{2}$ \\
$\ldots$ & $\ldots$ & $\ldots$ & $\ldots$ & $\ldots$ & $\ldots$ \\
$\mathrm{R}$ & $\mathrm{X}_{\mathrm{r} 1}$ & $\mathrm{X}_{\mathrm{r} 2}$ & $\ldots$ & $\mathrm{X}_{\mathrm{rc}}$ & $\mathrm{R}_{\mathrm{r}}$ \\
$\begin{array}{c}\text { Column } \\
\text { total }\end{array}$ & $\mathrm{C}_{1}$ & $\mathrm{C}_{2}$ & $\ldots$ & $\mathrm{C}_{\mathrm{c}}$ & Grand total \\
\hline
\end{tabular}


Recall that $X_{i j} \sim \mathrm{B}\left(1, \Delta_{j}\right)$, and $X_{i k}$ is independent of $X_{j k}$ for $i \neq j$ but $X_{i k}$ may be correlated with $X_{i l}$. Thus, the rows of Table 2 are independent random vectors. However, random variables in a row may possibly be correlated with $\mathrm{E}\left(X_{i j} * X_{i k}\right)$ $=\Delta_{j, k}$ where $j \neq k=1,2, \ldots, r$. For this setting, a randomization test was developed by Cochran (1950) which tests a more constrained null hypothesis, given as follows.

$$
H_{00}: \Delta_{1}=\Delta_{2}=\ldots=\Delta_{c} \text { and } \Delta_{i j}=\Delta_{1,2}=\Delta_{1,3}=\ldots=\Delta_{(c-l), c}
$$

where $\Delta_{l m}=\mathrm{P}\left(X_{i l}=1, X_{i m}=1\right), i=1,2, \ldots, r, l \neq m$.

Clearly, $H_{00}$ implies $H_{0}$ in (1). But the converse does not hold. Note that $H_{00}$ is equivalent to

$$
{ }^{*} H_{00}: \Delta_{l}=\Delta_{2}=\ldots=\Delta_{c} \text { and } \omega_{i j}=\omega_{1,2}=\omega_{1,3}=\ldots=\omega_{(c-l), c}
$$

where $\omega_{l m}=\operatorname{corr}\left(\mathrm{X}_{\mathrm{i}}, \mathrm{X}_{\mathrm{im}}\right), l \neq m$.

The second condition in equation (2) is commonly called "compound symmetry" (Gayle, 2010). Mandansky (1963) called $H_{00}$ the hypothesis of interchangeability. Also, Berger and Gold (1973), and Bhapker and Somes (1977) showed that the CQT statistic has a restrictive $\chi^{2}$ distribution only under ${ }^{*} H_{00}$ or $H_{00}$.

\subsection{Cochran's Q-statistic}

The CQT statistic for binary responses, $X_{i, j}$ in $c$ matched groups from $r$ subjects, is computed as

$$
Q=\frac{c(c-1) \sum_{j=1}^{c}\left(G_{j}-\bar{G}\right)^{2}}{c \sum_{i=1}^{r} L_{i}-\sum_{i=1}^{r} L_{i}^{2}}
$$

where $Q$ is distributed approximately as chi square with $d f=c-1, G_{j}$ is the total number of "successes" in the $j^{\text {th }}$ column. $\bar{G}$ is the mean of the $G_{j}$, and $L_{i}$ is the total number of "successes" in the $i^{\text {th }}$ row. 
A formula which is equivalent and easily derivable, but which simplifies computation, is

$$
Q=\frac{(c-1)\left[c \sum_{j=1}^{c} G_{j}^{2}-\left(\sum_{j=1}^{c} G_{j}\right)^{2}\right]}{c \sum_{i=1}^{r} L_{i}-\sum_{i=1}^{r} L_{i}^{2}} .
$$

For 'large' samples, the test statistic $Q$ is distributed as $\chi^{2}$ with $c-1$ degrees of freedom. As in the McNemar test, only subjects that do not have the same response in all categories contribute to the overall $Q$ statistic. The p-value for the test is computed as:

$$
p \text {-value }=\operatorname{Pr}\left(Q>\chi_{1-\alpha, c-1}^{2}\right)
$$

where $\chi_{1-\alpha, c-1}^{2}$ is the value of the $(1-\alpha)$ quartile of the $\chi^{2}$ distribution with $c-1$ degrees of freedom.

When $c=2$, the CQT becomes equivalent to the well-known McNemar's test (McNemar, 1947). Therefore, the rows are viewed as independent realizations of random variables $\left(X_{1}, X_{2}\right)$, where $X_{j}=1$ if the response to condition $j$ is a success and 0 otherwise, $j=1,2$. A summary of the entries is presented in a $2 \times 2$ table in Table 3.

Table 3. Summary counts for two proportions comparison

\begin{tabular}{ccccc}
\hline & & $X_{2}$ & & \\
\hline$X_{1}$ & 0 & & 1 & Total \\
0 & $\mathrm{e}$ & $\mathrm{f}$ & $\mathrm{e}+\mathrm{f}$ \\
1 & $\mathrm{~g}$ & $\mathrm{~h}$ & $\mathrm{~g}+\mathrm{h}$ \\
Total & $\mathrm{e}+\mathrm{g}$ & $\mathrm{f}+\mathrm{h}$ & $\mathrm{N}$ \\
\hline
\end{tabular}

McNemar's test statistic where $c=2$ is given as

$$
\chi^{2}=\frac{(f-g)^{2}}{f+g} \text {. }
$$

$H_{0}: \Delta_{1} \equiv P\left(X_{1}=1\right)=P\left(X_{2}=1\right) \equiv \Delta_{2}$ vs $H_{1}: P\left(X_{1}=1\right) \neq P\left(X_{2}=1\right)$. 
$H_{0}$ is rejected at the appropriate level of significance $\alpha$ if $\chi^{2}>\chi_{(1-\alpha), 1}^{2}$.

Note that this test statistic uses only those blocks in which a unit responds differently to the two environments. This makes sense, since these are the only blocks that contain information about $\Delta_{1}-\Delta_{2}$. By default, compound symmetry holds since $c=2$.

\subsection{Relationship between McNemar's test and Cochran's Q-test}

McNemar's test and the CQT are equivalent when there are $c=2$ responses in each row of Table 2. To verify this statement, we start out by setting $c=2$ in $Q$; we have

$$
Q=\frac{2 \sum_{j=1}^{2}\left(G_{j}-\bar{G}\right)^{2}}{2 \sum_{i=1}^{r} L_{i}-\sum_{i=1}^{r} L_{i}^{2}}
$$

and the numerator in equation (4) can be expressed as follows:

$$
\begin{aligned}
2 \sum_{j=1}^{2}\left(G_{j}-\bar{G}\right)^{2} & =2\left[\left(G_{l}-\left(G_{l}+G_{2}\right) / 2\right)^{2}+\left(G_{2}-\left(G_{l}+G_{2}\right) / 2\right)^{2}\right] \\
& =\left(G_{l}-G_{2}\right)^{2}=(g+h-f-h)^{2}=(f-g)^{2} .
\end{aligned}
$$

Similarly,

$$
2 \sum_{i=1}^{r} L_{i}-\sum_{i=1}^{r} L_{i}^{2}=2(f+g+2 h)-(f+g+4 h)=(\mathrm{f}+\mathrm{g}) .
$$

Dividing equation (5) by (6) gives the desired result of equation (3).

\subsection{Mean comparison}

When the outcome of the CQT indicates that the null hypothesis of equality of success proportions is to be rejected, it is often necessary to proceed to determine which of the groups are different by computing multiple pairwise comparisons. Pairwise tests between groups "a" and " $b$ " test the null hypothesis

$$
H_{0}: \Delta_{l}=\Delta_{2}=\ldots=\Delta_{c} \text { vs } H_{l}: \Delta_{i} \neq \Delta_{j} \text { for at least one pair }(i, j) \text {. }
$$


Sheskin (2011) described two methods: the minimum required difference (MRD) method, and pairwise McNemar tests among groups. Both methods for pairwise comparison use the Bonferroni alpha adjustment to control the overall experiment-wise error rate of the tests. The adjustment simply divides the overall required alpha, $\alpha$, by the number of pairwise tests, $c$, where

$$
v=\frac{c(c-1)}{2} \text {. }
$$

The alpha-level for each individual test, $\alpha_{a d j}$, is

$$
\alpha_{a d j}=\frac{\alpha}{v} .
$$

For this research, the MRD is adopted. This is because it is more robust, since it uses all of the available information in the data in its calculations, while the multiple McNemar tests comparison method uses only the values from subjects that have different responses for the two categories, not all of the data. Hence, the MRD is more powerful for finding the difference among pairs than the McNemar test (Berger and Gold, 1973).

For sufficiently large sample sizes (i.e. $n \geq 4$ and $n c \geq 24$, where $n$ is the number of subjects for which the responses are not all 0's or 1's), the minimum required difference in proportions for any pair of the $c$ experimental groups to be declared different is

$$
M R D=z_{a d j} \sqrt{2\left[\frac{c \sum_{i=1}^{r} L_{i}-\sum_{i=1}^{r} L_{i}^{2}}{r^{2} c(c-1)}\right]}
$$

where all parameters are defined as in the CQT statistic, and zadj is the value of the quantile, that is, $1-\alpha_{a d j} / 2$ from the standard normal distribution.

Two groups are confirmed to be significantly different with protected overall alpha, $\alpha$, if their absolute difference in proportions is greater than $M R D$, that is, if $\left|\Delta_{\mathrm{a}}-\Delta_{\mathrm{b}}\right|>M R D$. 


\section{Results and discussion}

Table 4 below presents the number and proportion of negatives and positives for the nine geohelminth egg types from 184 soil samples taken from the 16 different sampled parks in Abuja metropolis. MedCalc version 19.2.1 software was used for data analysis.

Table 4. Frequency distribution of geohelminth eggs

\begin{tabular}{ccccc}
\hline Geohelminth eggs & $\begin{array}{c}\text { Negative } \\
\text { cases }\end{array}$ & $\begin{array}{c}\text { Positive } \\
\text { cases }\end{array}$ & $\begin{array}{c}\text { Negative } \\
\text { proportion }(\%)\end{array}$ & $\begin{array}{c}\text { Positive } \\
\text { proportion }(\%)\end{array}$ \\
\hline Ascaris & 172 & 12 & 93.48 & 6.52 \\
Coccidia & 137 & 47 & 74.46 & 25.54 \\
Hookworm & 166 & 18 & 90.22 & 9.78 \\
Mite & 161 & 23 & 87.5 & 12.5 \\
Stongylus & 172 & 12 & 93.48 & 6.52 \\
Strongyloides & 183 & 1 & 99.46 & 0.54 \\
Taenia & 135 & 49 & 73.37 & 26.63 \\
Toxocara & 157 & 27 & 85.33 & 14.67 \\
Trichuris & 160 & 24 & 86.96 & 13.04 \\
\hline
\end{tabular}

It can be observed that in all of the 184 soil samples from the 16 recreational parks, Taenia and Coccidia have the largest proportions of positives $(26.33 \%$ and

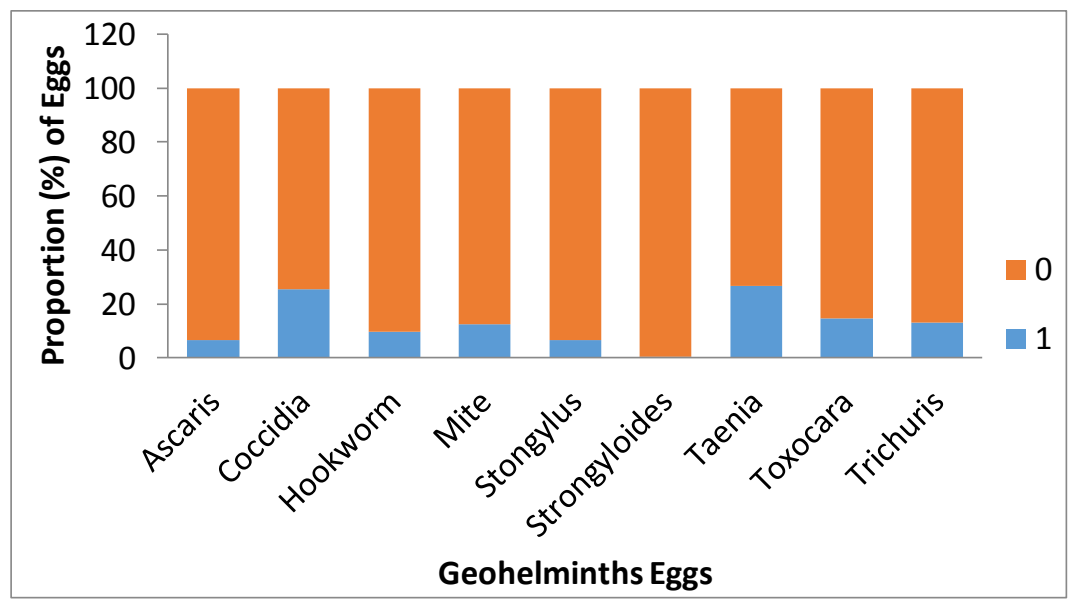

Figure 1. Positive (1) and negative (0) proportions (\%) of geohelminth eggs 
$25.54 \%$ respectively) amongst all geohelminth egg types. The proportion of Strongyloides eggs is the lowest, at $0.54 \%$. Figure 1 presents a bar chart of positive and negative proportions of all nine geohelminth egg types.

Table 5 presents the CQT result for comparison of the proportions of Ascaris, Coccidia, hookworm, mite, Strongylus, Strongyloides, Taenia, Toxocara and Trichuris. The null hypothesis for the test is as follows.

$\mathbf{H}_{\mathbf{0}}$ : the proportions of positive geohelminth eggs in all parks from 184 soil samples are equal.

$\mathbf{H}_{\mathbf{1}}$ : the proportions of positive geohelminth eggs in at least one sampled park from 184 soil samples are different.

Table 5. Cochran's Q-test result

\begin{tabular}{cc}
\hline Number of parks & 16 \\
Number of samples, $n$ & 184 \\
Cochran's Q & 94.8706 \\
Degree of freedom & 8 \\
P-value & 0.0001 \\
\hline
\end{tabular}

From Table 5 above it can be observed that at a 5\% level of significance we reject our stated null hypothesis, because the p-value of 0.0001 is below 5\%. Therefore, it can be concluded that the proportions of geohelminth eggs in the 184 soil samples from 16 recreational parks in Abuja metropolis are different.

Based on the CQT result, which showed a significant difference among the proportions of geohelminth eggs, the results of a mean comparison test using the MRD method are presented in Table 6 . The proportions of the nine geohelminth egg types are compared to determine which has the highest significant proportion.

The multiple mean comparison result in Table 6 indicates that the proportions of Ascaris, Coccocidia and Taenia eggs are significantly different from each other, with absolute differences of $19.02 \%$ and $20.11 \%$ respectively. Also, the proportion of Coccocidia eggs is significantly different from the proportions of hookworm, mite, Strongylus, Strongyloides and Trichuris eggs, with absolute 
Table 6. Pairwise comparison of the nine soil egg types

\begin{tabular}{|c|c|c|c|c|c|}
\hline Comparison & $\Delta_{i}$ & $\Delta_{j}$ & $\left|\Delta_{\mathrm{i}}-\Delta_{\mathrm{i}}\right|$ & MRD & Decision \\
\hline Ascaris - Coccidia & 6.52 & 25.54 & 19.02 & 11.33 & YES \\
\hline Ascaris - Hookworm & 6.52 & 9.78 & 3.26 & 11.33 & NO \\
\hline Ascaris - Mite & 6.52 & 12.5 & 5.98 & 11.33 & NO \\
\hline Ascaris - Strongylus & 6.52 & 6.52 & 0 & 11.33 & NO \\
\hline Ascaris - Strongyloides & 6.52 & 0.54 & 5.98 & 11.33 & NO \\
\hline Ascaris - Taenia & 6.52 & 26.63 & 20.11 & 11.33 & YES \\
\hline Ascaris - Toxocara & 6.52 & 14.67 & 8.15 & 11.33 & NO \\
\hline Ascaris - Trichuris & 6.52 & 13.04 & 6.52 & 11.33 & NO \\
\hline Coccidia - Hookworm & 25.54 & 9.78 & 15.76 & 11.33 & YES \\
\hline Coccidia - Mite & 25.54 & 12.5 & 13.04 & 11.33 & YES \\
\hline Coccidia - Strongylus & 25.54 & 6.52 & 19.02 & 11.33 & YES \\
\hline Coccidia - Strongyloides & 25.54 & 0.54 & 25 & 11.33 & YES \\
\hline Coccidia - Taenia & 25.54 & 26.63 & 1.09 & 11.33 & NO \\
\hline Coccidia - Toxocara & 25.54 & 14.67 & 10.87 & 11.33 & NO \\
\hline Coccidia - Trichuris & 25.54 & 13.04 & 12.5 & 11.33 & YES \\
\hline Hookworm - Mite & 9.78 & 12.5 & 2.72 & 11.33 & NO \\
\hline Hookworm - Strongylus & 9.78 & 6.52 & 3.26 & 11.33 & NO \\
\hline Hookworm - Strongyloides & 9.78 & 0.54 & 9.24 & 11.33 & NO \\
\hline Hookworm - Taenia & 9.78 & 26.63 & 16.85 & 11.33 & YES \\
\hline Hookworm - Toxocara & 9.78 & 14.67 & 4.89 & 11.33 & NO \\
\hline Hookworm - Trichuris & 9.78 & 13.04 & 3.26 & 11.33 & NO \\
\hline Mite - Strongylus & 12.5 & 6.52 & 5.98 & 11.33 & NO \\
\hline Mite - Strongyloides & 12.5 & 0.54 & 11.96 & 11.33 & YES \\
\hline Mite - Taenia & 12.5 & 26.63 & 14.13 & 11.33 & YES \\
\hline Mite - Toxocara & 12.5 & 14.67 & 2.17 & 11.33 & NO \\
\hline Mite - Trichuris & 12.5 & 13.04 & 0.54 & 11.33 & NO \\
\hline Strongylus - Strongyloides & 6.52 & 0.54 & 5.98 & 11.33 & NO \\
\hline Strongylus - Taenia & 6.52 & 26.63 & 20.11 & 11.33 & YES \\
\hline Strongylus - Toxocara & 6.52 & 14.67 & 8.15 & 11.33 & NO \\
\hline Strongylus - Trichuris & 6.52 & 13.04 & 6.52 & 11.33 & NO \\
\hline Strongyloides - Taenia & 0.54 & 26.63 & 26.09 & 11.33 & YES \\
\hline Strongyloides - Toxocara & 0.54 & 14.67 & 14.13 & 11.33 & YES \\
\hline Strongyloides - Trichuris & 0.54 & 13.04 & 12.5 & 11.33 & YES \\
\hline Taenia-Toxocara & 26.63 & 14.67 & 11.96 & 11.33 & YES \\
\hline Taenia-Trichuris & 26.63 & 13.04 & 13.59 & 11.33 & YES \\
\hline Toxocara-Trichuris & 14.67 & 13.04 & 1.63 & 11.33 & NO \\
\hline
\end{tabular}

YES means an absolute difference value greater than the MRD value, which implies significant difference

differences of $15.76 \%, 13.04 \%, 19.02 \%$ and $25 \%$. At an absolute difference of $16.85 \%$, the proportion of hookworm eggs is significantly different from the proportion of Taenia eggs, while the proportion for mites is significantly different 
from the proportions of Strongyloides and Taenia eggs, with absolute differences of $11.96 \%$ and $14.13 \%$ respectively. The proportions of Strongylus and Taenia eggs are significantly different from each other, with an absolute difference of $20.11 \%$. The proportions of Taenia, Toxocara and Trichuris eggs in the soil are significantly larger than the proportion of Strongyloides eggs, with absolute differences of $26.09 \%, 14.13 \%$ and $12.5 \%$ respectively. Finally, it was observed that the proportion of Taenia eggs is significantly larger than the proportions of Toxocara and Trichuris eggs, with absolute differences of $11.96 \%$ and $13.59 \%$ respectively.

\section{Conclusions}

This research studied the prevalence of geohelminth eggs in 184 soil samples from 16 recreational parks in Abuja metropolis, Nigeria. The CQT was applied to determine whether there was a significant difference in the proportions of the studied egg types in the soil samples, and it was determined which of the egg types had significantly larger proportions using the MRD technique. It was found that the geohelminth eggs occur in significantly different proportions in the 184 soil samples examined. Also, Taenia and Coccocidia eggs are highly prevalent, with proportions significantly larger than the proportions of the other geohelminth eggs in the examined soil samples, based on the MRD mean comparison test.

\section{REFERENCES}

Adegnika A.A., Agnandji S.T., Chai S.K., Ramharter M., Breitling L., Kendjo E., Issifou S., Yazdanbakhsh M., Kombila M., Kremsner P.G. (2007): Increased prevalence of intestinal helminth infection during pregnancy in a Sub-Saharan African community. Wien. Klin. Wochenschr 119: 712-716.

Aoyama H., Hirata T., Sakugawa H., Watanabe T., Miyagi S., Maeshiro T., Chinen T., Kawane M., Zaha O., Nakayoshi T., Kinjo F., Fujita J. (2007): An inverse relationship between autoimmune liver diseases and strongyloides stercoralis infection. Am. J. Trop. Med. Hyg. 76(5): 972-976.

Berger A., Gold R. (1973): Note on Cochran's Q Test for the Comparison of Correlated Proportions. J. Am. Stat. Assoc. 68: 989-993. 
Bethony J., Brooker S., Albonico M., Geiger S.M., Loukas A., Diemert D., Hotez P.J. (2006): Soil-transmitted helminth infections: Ascariasis, trichuriasis, and hookworm. Lancet 367: 1521-1532.

Bhapkar V.P., Somes G.W. (1977): Distribution of Q When Testing Equality of Matched Proportions. J. Am. Stat. Assoc. 72: 658-661.

Cochran W.G. (1950): The comparison of percentages in matched samples. Biometrika 37: 256-266.

Flohr C., Quinnell R.J., Britton J. (2009): Do helminth parasites protect against atopy and allergic disease? Clin. Exp. Allergy. 39(1): 20-32.

Galaktionov K.V. (2017): Patterns and processes influencing helminth parasites of Arctic coastal communities during climate change. J. Helminthol. 91(4): 387-408.

Gayle S.S. (2010): A simulation study of the size and power of Cochran's Q versus the standard Chi-square test for testing the equality of correlated proportions. An M.Sc Report. Kansas State University Manhattan, Kansas, USA.

Goodman D., Haji H.J., Bickle Q.D., Stoltzfus R.J., Tielsch J.M., Ramsan M., Savioli L., Albonico M.A. (2007): Comparison of methods for detecting the eggs of ascaris, trichuris, and hookworm in infant stool, and the epidemiology of infection in zanzibari infants. Am. J. Trop. Med. Hyg. 76: 725-731.

Isaac C., Turay P.N., Inegbenosun C.U., Ezekiel S.A., Adamu H.O., Ohiolei J.A. (2019): Prevalence of soil-transmitted helminths in primary school playgrounds in Edo State, southern Nigeria. Helminthologia 56(4): 282-295.

Karshima S.N. (2018): Prevalence and distribution of soil-transmitted helminth infections in Nigerian children: a systematic review and meta-analysis. Infect. Dis. Poverty., https://doi.org/10.1186/s40249-018-0451-2

Khana J.S., Provenchera J.F., Forbesb M.R., Malloryc M.L., Lebarbenchond C., McCoye K.D. (2019): Parasites of seabirds: A survey of effects and ecological implications. Adv. Mar. Biol., https://doi.org/10.1016/bs.amb.2019.02.001

Lammie P.J., Fenwick A., Utzinger J. (2006): A blueprint for success: Integration of neglected tropical disease control programmes. Trends Parasitol. 22: 313-321.

Lindquist H.D.A. Cross J.H. (2017): Helminths. In: Cohen, J., Powderly, W. G., and Opal S.M. (Eds.). Infectious Diseases, $4^{\text {th }}$ ed., Vol II, 2017, p. 1763-1779. https://doi:10.1016/B978-0-7020-6285-8.00195-7

Lustigman S., Prichard R.K., Gazzinelli A., Grant W.N., Boatin B.A., McCarthy J.S., Basáñez M.G. (2012): A Research Agenda for Helminth Diseases of Humans: The Problem of Helminthiases. PLoS Negl Trop Dis., https://doi.org/10.1371/ journal.pntd.0001582

Madansky A. (1963): Tests of Homogeneity for Correlated Samples. J. Am. Stat. Assoc. 58: 97-119.

Maikai B.V., Umoh J.U., Ajanusi O.J., Ajogi I. (2008): Public health implications of soil contaminated with helminth eggs in the metropolis of Kaduna, Nigeria. J. Helminthol. 82: 113-118.

McNemar Q. (1947): Note on the sampling error of the difference between correlated proportions or percentages. Psychometrika. 12(2): 153-157.

Ngwese M.M., Manouana P.G., Moure N.A.P., Ramharter M., Esen M., Adégnika A.A. (2020): Diagnostic Techniques of Soil-Transmitted Helminths: Impact on Control Measures. Trop. Med. Infect. Dis., https://doi:10.3390/tropicalmed5020093 
Okeh U.M., Oyeka I.C.A., Igwenagu C. (2016): An alternative approach to Cochran Q test for dichotomous data. MOJ Pub. Health. 4(4): 114-117.

Page A.L., Miller R.H., Keeney D.R. (1982): Methods of Soil Analysis Part 2: Chemical and Microbial Properties. Madison, Wisconsin: USA.

Pal A. Chakravarty A.K. (2020): Genetics and Breeding for Disease Resistance of Livestock, $1^{\text {st }}$ ed. Academic Press, USA, p. 193-208. https://doi.org/10.1016/B9780-12-816406-8.00013-9

Pereira T.V., Patsopoulos N.A., Salanti G., Loannidis J.P.A. (2010): Critical interpretation of Cochran's $Q$ test depends on power and prior assumptions about heterogeneity. Res. Synth. Methods. 1: 149-161.

Ruiz de Ybanez M.R., Garijo M.M., Goyena M., Alonso F. (2000): Improved methods for recovering eggs of Toxocara canis from soil. J. Helminthol. 74: 349-353.

Sheskin D. (2011): Handbook of Parametric and Nonparametric Statistical Procedures, $5^{\text {th }}$ ed. Chapman \& Hall/CRC, London.

Soulsby E.J.L. (1987): Larva Migrans in Perspective. In: Geerts S., Kumar V. and Brandt J. (eds) Helminth Zoonoses. Current Topics in Veterinary Medicine and Animal Science, Vol XLIII. Springer, Dordrecht, https://doi.org/10.1007/978-94-0093341-5_20

Vickers A.J. (2005): Parametric versus non-parametric statistics in the analysis of randomized trials with non-normally distributed data. BMC Med. Res. Methodol., https://doi.org/10.1186/1471-2288-5-35

Vonk J. (2011): Methodology and Statistics for Linguistic Research. Cochran's Q test. Available at: http://www.let.rug.nl/nerbonne/teach/rema-stats-methseminar/ presentations/Vonk-Cochrans-Q-2011-June-7.pdf, assessed October, 2020.

World Health Organisation [WHO] (2002): Prevention and Control of Schistosomiasis and Soil-Transmitted Helminths: Report of a WHO Expert Committee; Geneva Technical Report Series; World Health Organisation: Geneva, Switzerland, pp. 1-57. 